\title{
Performance analysis and power allocation for multi-hop multi-branch amplify-and-forward cooperative networks over generalized fading channels
}

\author{
Mohammadali Mohammadi", Mehrdad Ardebilipour, Zahra Mobini and Ramezan-Ali Sadegh Zadeh
}

\begin{abstract}
In this article, efficient power allocation strategies for multi-hop multi-branch amplify-and-forward networks are developed in generalized fading environments. In particular, we consider the following power optimization schemes: (i) minimizing of the all transmission powers subject to an outage constraint; and (ii) minimizing the outage probability subject to constraint on total transmit powers. In this study, we first derive asymptotically tight approximations for the statistics of the received signal-to-noise ratio (SNR) in the system under study with maximal ratio combining and selection combining receiver. With the statistical characterization of the received SNR, we then carry out a thorough performance analysis of the system. Finally, the asymptotic expression of the outage probability is used to formulate the original optimization problems using geometric programming (GP). The GP can readily be transformed into nonlinear convex optimization problem and therefore solved efficiently and globally using the interior-point methods. Numerical results are provided to substantiate the analytical results and to demonstrate the considerable performance improvement achieved by the power allocation.
\end{abstract}

\section{Introduction}

Recently, it has been shown that the throughput, coverage, and battery life of resource-constrained wireless ad hoc networks can be increased through the use of multi-hop relay transmission $[1,2]$. The main idea is that communication is achieved by relaying the information from the source to the destination through the use of many intermediate terminals in between. In a multi-hop multibranch transmission system, a source communicates with the destination through several multi-hop branches, each of which consists of multiple intermediate relay nodes. As a result, the destination node can receive multiple independent copies of the same signal and can achieve diversity without the need to install multiple antennas at the source node or the destination node. On the other hand, emerging wireless applications, e.g., wireless sensor and ad hoc networks, have an increasing demand for small devices having limited battery lifetimes. For a more

\footnotetext{
*Correspondence: m.a.mohammadi@ee.kntu.ac.ir

Department of Electrical Engineering, K. N. Toosi University of Technology, Tehran, Iran
}

efficient use of the power resources, the problem of optimally distributing the power among the source node and the relay nodes has drawn great attention from wireless service providers and academia.

Over the past decade, a considerable effort in the literature has been devoted to the performance analysis of cooperative relay systems. In particular, the performance of dual-hop amplify and forward (AF) networks has widely been analyzed in [3-5] and [6-10] for single and multiple relay scenarios, respectively. Multi-hop multi-branch AF network has also been investigated in a few recent works [11-14]. Specifically, Ribeiro et al. [11] studied the symbol error probability of these networks for a class of fading models, whose probability density functions (pdf) have non-zero values at the origin, including Rayleigh and Rician fading channels, when the average signal-to-noise ratio (SNR) is sufficiently large. Renzo et al. [13] exploit the Moment Generating Function (MGF)-based approach for performance analysis of multi-hop multi-branch networks over fading channels.

\section{照 Springer}

(c) 2013 Mohammadi et al.; licensee Springer. This is an Open Access article distributed under the terms of the Creative Commons Attribution License (http://creativecommons.org/licenses/by/2.0), which permits unrestricted use, distribution, and reproduction in any medium, provided the original work is properly cited. 
Amarasuriya et al. [14] proposed a new class of upper bounds on the end-to-end SNR of a multi-hop system and then derived an asymptotic expression for the symbol error rate (SER) of the multi-hop multi-branch set-up in independent and identically distributed (i.i.d.) Nakagami- $m$ fading conditions. Common to all aforementioned studies is the simplified assumption that all hops and also all diversity paths have the same fading conditions. However, due to the wide spatial distribution of the relay nodes in a practical wireless system, the hops may undergo different kinds of fading conditions. In the ensuing text, we refer to this set-up as generalized fading environments. Note, moreover, that resource allocation is assumed to be fixed in these works. In other words, all theses works provide complicated bounds on the performance metrics such as outage probability [11], SER [14], or numerical methods [13], which render the practical solutions for the resource allocation problem impossible.

Management of available radio resources plays a key role in improving the performance of wireless networks. Many research efforts have been devoted to investigate the performance improvement of relay networks by optimally allocate the radio resources [15-17]. It is worth mentioning that dual-hop relaying scheme is typically considered in the aforementioned studies and power-optimized multi-hop relaying is only studied in [17]. To the best of the authors' knowledge, the ultimate benefit of power control in multi-hop multi-branch networks has not been studied in the existing literature. One main goal of this article is to fill this important gap.

In this article, we develop efficient power allocation frameworks for multi-hop multi-branch networks in generalized fading environments. In particular, our power allocation schemes aimed at: (i) minimizing the transmitter powers subject to an outage constraint; and (ii) minimizing the outage probability subject to constraint on total transmit powers. Thanks to the asymptotically tight approximation of the outage performance, that we develop for both maximal ratio combining (MRC) and selection combining $(\mathrm{SC})$ receivers, we can formulate the original optimization problems using geometric programming (GP). The GP can readily be transformed into nonlinear convex optimization problem and therefore solved efficiently and globally using the interior-point methods $[18,19]$.

The remainder of this article is organized as follows. Section 2 describes the system model. Section 3 studies the asymptotic performance evaluation of the multi-hop system. Section 4 presents the asymptotic analysis of the multi-hop multi-branch system. The problem formulation for power optimization is given in Section 5. Simulation and numerical results are presented in Section 6, followed by the conclusions in Section 7 .

\section{System model}

Consider a generalized cooperative system with $M$ diversity branches and $\left\{N_{i}\right\}_{i=1}^{M}$ hops for each branch as shown in Figure 1. We denote $R_{i, j}\left(1 \leq i \leq M\right.$ and $\left.1 \leq j \leq N_{i}-1\right)$ as the $j$ th relay in the $i$ th branch and $h_{i n}\left(1 \leq n \leq N_{i}\right)$ as the channel coefficient for the $n$th hop in the $i$ th branch. We assume that the distance between relay clusters (hop) is much larger than the distance between the nodes in any one cluster. Therefore, the channel gains of the hops are independently but not necessarily identically distributed (i.n.i.d).

When AF relaying is employed, the relay node $R_{i, j}$ amplifies the signal received from the preceding terminal by a factor $A_{i j}$ given by

$$
\left(A_{i j}\right)^{2}=\frac{P_{i j}}{P_{i(j-1)}\left|h_{i j}\right|^{2}+\sigma_{n}^{2}}, \quad 1 \leq i \leq M, \quad 1 \leq j \leq N_{i}-1,
$$

where $\sigma_{n}^{2}$ is the power of additive white Gaussian noise $(\mathrm{AWGN})^{\mathrm{a}}$ and $P_{i 0}$ is the source transmission power in branch $i$. Let we denote the instantaneous SNR of the $j$ th hop of the $i$ th branch by $\gamma_{i j}=\left|h_{i j}\right|^{2} P_{i(j-1)} / \sigma_{n}^{2}$. The received SNR of the $i$ th branch is given by [20]

$$
\gamma_{i}=\left[\prod_{j=1}^{N_{i}}\left(1+\frac{1}{\gamma_{i j}}\right)-1\right]^{-1},
$$

which can be well approximated by [20]

$$
\gamma_{i} \approx\left[\prod_{j=1}^{N_{i}}\left(\frac{1}{\gamma_{i j}}\right)\right]^{-1}
$$

especially for sufficiently large values of SNR.

While a number of different distributions are possible for fading amplitudes, we choose here the generalized Gamma distribution, whose pdf is [21]

$$
f_{h}(x)=\frac{2 v x^{2 v m-1}}{\beta^{2 v m} \Gamma(m)} \exp \left[-\left(\frac{x}{\beta}\right)^{2 v}\right],
$$

where $\Gamma(\cdot)$ is the gamma function defined in $[22$, Eq.(8.310.1)], $m$ is the fading parameter, $v$ is the shape parameter and $\Omega:=\beta^{v} m$ is the power-scaling parameter. In what follows, we will use the shorthand notation $X \sim$ $\mathcal{G}(a, b)$ to denote that $X$ follows generalized Gamma distribution with parameters $a$ and $b$. With a proper choice of three parameters $m, \beta$ and $v$ the generalized Gamma distribution can represent a wide variety of distributions including the Rayleigh $(m=v=1)$, Nakagami- $m(v=1)$, Weibull $(m=1)$, log-normal $(m \rightarrow \infty, v=0)$, and AWGN $(m \rightarrow \infty, v=1)$ cases. We also mention that although the Rician pdf cannot exactly be represented by a generalized Gamma, it indeed constitutes a very good 


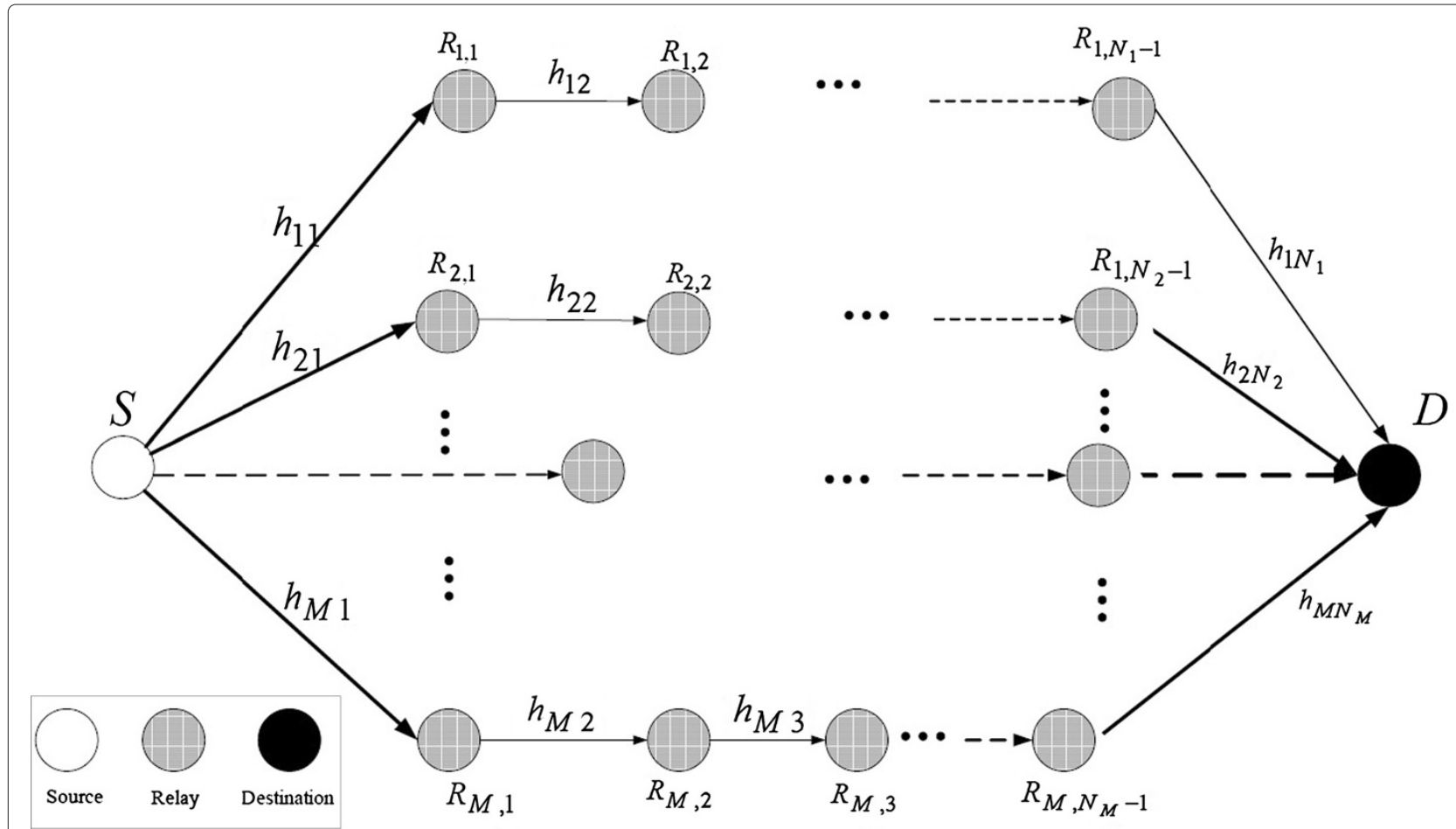

Figure 1 System model. Generic cooperative (multi-hop multi-branch) wireless system.

approximation if the shape parameter $v=1$ and the relationship $m \approx \frac{(K+1)^{2}}{2 K+1}$ between the Rician factor $K$ and the fading figure $m$ holds [23]. The pdf of $\gamma_{i j}$ then can be expressed as [24]

$$
f_{\gamma_{i j}}(\gamma)=\left(\frac{\xi_{i j}}{\bar{\gamma}_{i j}}\right)^{m_{i j} v_{i j}} \frac{v_{i j}}{\Gamma\left(m_{i j}\right)} \gamma^{v_{i j} m_{i j}-1} \exp \left[-\left(\frac{\xi_{i j}}{\bar{\gamma}_{i j}} \gamma\right)^{v_{i j}}\right] \text {, }
$$

where $\xi_{i j}=\Gamma\left(m_{i j}+1 / v_{i j}\right) / \Gamma\left(m_{i j}\right)$ and $\bar{\gamma}_{i j}=$ $\mathbb{E}\left\{\left|h_{i j}\right|^{2}\right\} P_{i(j-1)} / \sigma_{n}^{2}$, with $\mathbb{E}\{\cdot\}$ being the expectation operator.

\section{Asymmetric multi-hop system}

In this section, we study the performance of the asymmetric multi-hop systems. We first derive the asymptotic statistics of the received SNR at the destination. Then, we obtain closed-form expressions for the outage probability and the average SER of the system under the high SNR assumption.

\subsection{Statistics of the end-to-end SNR}

To analyze the performance of the multi-hop system, we need statistical characterization of its end-to-end SNR. In this section, we derive the cumulative distribution function (cdf), pdf and MGF of the received SNR.

Although the expression given in (3) for $\gamma_{i}$ is more mathematically tractable than the one given in (3), the statistics of $\gamma_{i}$ in (3) are unknown for an arbitrary number of hops. ${ }^{\text {b }}$ In order to keep a tractable analysis, we use the upper bound of $\gamma_{i}$ in (3) as [25],

$$
\gamma_{i} \leq \bar{\gamma}_{i}=\min \left(\gamma_{i 1}, \ldots, \gamma_{i N_{i}}\right), \quad i=1, \ldots M
$$

The following proposition summarizes the results on statistics of $\gamma_{i}$ in the high-SNR regime.

Proposition 1. Let $\gamma_{i j} \sim \mathcal{G}\left(m_{i j},\left(\frac{\bar{\gamma}_{i j}}{\xi_{i j}}\right)^{v_{i j}}\right), j=1, \ldots, N_{i}$, be the independent hop SNRs for the ith branch. The asymptotic cdf of $\overline{\gamma_{i}}$ is then given by

$$
\begin{aligned}
P_{\overline{\gamma_{i}}}(\gamma) \approx & \sum_{j=1}^{N_{i}} \frac{1}{\Gamma\left(m_{i j}+1\right)}\left(\frac{\Gamma\left(m_{i j}+1 / v_{i j}\right)}{\Gamma\left(m_{i j}\right)}\right)^{v_{i j} m_{i j}} \\
& \times\left(\frac{\gamma}{\bar{\gamma}_{i j}}\right)^{v_{i j} m_{i j}}+o\left(\bar{\gamma}_{i j}^{-\left(v_{i j} m_{i j}+1\right)}\right) .
\end{aligned}
$$

The pdf and the MGF of $\overline{\gamma_{i}}$ are, respectively, given by

$$
\begin{aligned}
p_{\overline{\gamma_{i}}}(\gamma) \approx & \sum_{j=1}^{N_{i}} \frac{v_{i j}}{\Gamma\left(m_{i j}\right)}\left(\frac{\Gamma\left(m_{i j}+1 / v_{i j}\right)}{\Gamma\left(m_{i j}\right)}\right)^{v_{i j} m_{i j}} \frac{1}{\bar{\gamma}_{i j}} \\
& \left(\frac{\gamma}{\bar{\gamma}_{i j}}\right)^{v_{i j} m_{i j}-1}+o\left(\bar{\gamma}_{i j}^{-\left(v_{i j} m_{i j}\right)}\right),
\end{aligned}
$$




$$
\begin{aligned}
\mathcal{M}_{\overline{\gamma_{i}}}(\gamma) \approx \sum_{j=1}^{N_{i}} \frac{\Gamma\left(v_{i j} m_{i j}+1\right)}{\Gamma\left(m_{i j}+1\right)} & \left(\frac{\Gamma\left(m_{i j}+1 / v_{i j}\right)}{\bar{\gamma}_{i j} \Gamma\left(m_{i j}\right)}\right)^{v_{i j} m_{i j}} \\
& \times\left(\frac{1}{s}\right)^{v_{i j} m_{i j}} .
\end{aligned}
$$

Proof. : See Proof of Proposition 1 in Appendix.

\subsection{Performance analysis}

With the statistical characterization of the received SNR derived in previous section, we can carry out a thorough performance analysis of the multi-hop system. We focus in what follows on outage probability and average SER performance measures.

\subsubsection{Outage probability}

The outage probability is one of the most commonly used performance measures in wireless systems. The outage probability in a multi-hop AF system is defined as the probability that the end-to-end instantaneous received SNR falls below a predetermined threshold $\gamma_{\text {th }}$. This threshold is a protection value of the SNR, above which the quality of service is satisfactory. Therefore, the outage probability is given by the $\operatorname{Pr}\left(\gamma_{i}<\gamma_{\text {th }}\right)$, which can easily be calculated by evaluating the cdf of $\gamma_{i}$ at $\gamma_{\text {th }}$. Consequently, asymptotic expression for the outage probability of the considered system over asymmetric fading channels can be obtained using (7) as

$$
\begin{aligned}
P_{\text {out }} \approx & \sum_{j=1}^{N_{i}} \frac{1}{\Gamma\left(m_{i j}+1\right)}\left(\frac{\Gamma\left(m_{i j}+1 / v_{i j}\right)}{\Gamma\left(m_{i j}\right)}\right)^{v_{i j} m_{i j}} \\
& \times\left(\frac{\gamma_{t h}}{\bar{\gamma}_{i j}}\right)^{v_{i j} m_{i j}}+o\left(\bar{\gamma}_{i j}^{-\left(v_{i j} m_{i j}+1\right)}\right) .
\end{aligned}
$$

For the special case of i.i.d Nakagami- $m$ fading where $m_{i j}=m_{i}, \bar{\gamma}_{i j}=\bar{\gamma}_{i}$, and $v_{i j}=1,(7)$ is reduced to

$$
P_{\mathrm{out}} \approx \frac{N_{i} m_{i}^{m_{i}}}{\Gamma\left(m_{i}+1\right)}\left(\frac{\gamma_{t h}}{\bar{\gamma}_{i}}\right)^{m_{i}}+o\left(\bar{\gamma}_{i}^{-\left(m_{i}+1\right)}\right)
$$

which is consistent with the result obtained in [14].

\subsubsection{SER}

In addition to the outage probability, the average SER, is another standard performance criterion of cooperative diversity systems. The derived MGF can be used to evaluate the average SER of the multi-hop AF system under $M$-PSK and $M$-QAM. The average SER of $M$-PSK can be written as [26]

$$
P_{e}=\frac{1}{\pi} \int_{0}^{(M-1) \pi / M} \mathcal{M}_{\bar{\gamma}_{i}}\left(\frac{g_{\mathrm{psk}}}{\sin ^{2} \theta}\right) d \theta
$$

where $g_{\text {psk }}=\sin ^{2}(\pi / M)$. For the square $M$-QAM signals that have constellation size $M=2^{k}$ with an even $k$, the average SER is given [26] as

$$
\begin{aligned}
P_{e}=\frac{4}{\pi} & \left(1-\frac{1}{\sqrt{M}}\right) \int_{0}^{\pi / 2} \mathcal{M}_{\bar{\gamma}_{i}}\left(\frac{g_{\mathrm{qam}}}{\sin ^{2} \theta}\right) d \theta \\
& -\frac{4}{\pi}\left(1-\frac{1}{\sqrt{M}}\right)^{2} \int_{0}^{\pi / 4} \mathcal{M}_{\bar{\gamma}_{i}}\left(\frac{g_{\mathrm{qam}}}{\sin ^{2} \theta}\right) d \theta,
\end{aligned}
$$

where $g_{\text {qam }}=3 / 2(M-1)$.

Closed-form solutions for (12) and (13) in the general case seem analytically intractable. ${ }^{\mathrm{c}}$ However, using the available software packages such as Mapel and Mathematica this evaluation can be performed easily for a required degree of accuracy. The numerical results and simulation results are discussed in Section 6.

\section{Asymmetric multi-hop multi-branch system}

In this section, we study the performance of the multihop multi-branch AF systems in generalized fading channels. The destination node combines the received signals from different paths. Specifically, we examine two different combining techniques: MRC and SC [26]. With MRC, the received signals from multiple diversity branches are cophased, weighted, and combined to maximize the output SNR. ${ }^{\mathrm{d}}$ MRC provides the maximum performance improvement relative to all other combining techniques by maximizing the SNR of the combined signal. However, MRC also has the highest complexity of all combining techniques since it requires knowledge of the fading amplitude in each signal branch. As such we consider MRC as an important theoretical benchmark to quantify the performance of the considered network. SC is often used in practice as an alternative technique because of its reduced complexity relative to the optimum MRC scheme. In its conventional form, SC diversity only processes one of the diversity branches, specifically, the one determined by the receiver to have the highest SNR. The most important reason behind the popularity of the SC is the simplicity in implementation and decrease in resource requirement and complexity at the receiver, while still achieving full diversity.

\subsection{Statistics of combined SNR}

To analyze the performance of MRC and SC, we need statistical characterization of their combined SNR. In this section, we derive the cdf, pdf, and MGF of the combined SNR with MRC and SC. 


\subsubsection{MRC}

MRC is the optimum combining scheme in the absence of interference [26, Ch .11]. The total SNR at the output of the MRC combiner is simply given by

$$
\gamma_{\mathrm{MRC}} \leq \bar{\gamma}_{\mathrm{MRC}}=\sum_{i=1}^{M} \bar{\gamma}_{i}
$$

To obtain the statistics of $\bar{\gamma}_{M R C}$, i.e., sum of several independent variables, we need the following lemma.

Lemma 1. Let us consider a finite set of an arbitrary and independent nonnegative random variables $(R V) X=$ $\left\{x_{1}, \ldots, x_{M}\right\}$, whose $p d f$ 's, $p_{x_{k}}(\cdot) k=1, \ldots, M$, tends to $p_{x_{k}}=a_{k} \gamma^{t_{k}}+o\left(\gamma^{t_{k}+\epsilon}\right)$ for $\gamma \rightarrow 0^{+}$and $\epsilon>0$. If the $R V$ $z_{k}$ is defined as

$$
z_{k} \triangleq x_{1}+x_{2}+\cdots+x_{k} \quad k=1, \ldots, M
$$

then the cdf of $z_{k}$ can be expressed as

$$
\begin{aligned}
P_{z_{k}}(\gamma) & =D_{k} \gamma^{\lambda_{k}}+o\left(\gamma^{\lambda_{k}+\epsilon}\right) \\
& =\left(\prod_{i=1}^{k} a_{i} \Delta_{i}\right) \gamma^{\lambda_{k}}+o\left(\gamma^{\lambda_{k}+\epsilon}\right), \\
\lambda_{i} & =\lambda_{i-1}+t_{i}+1=i+\sum_{\ell=1}^{i} t_{\ell}, \\
\Delta_{i} & =\frac{\Gamma\left(1+\lambda_{i-1}\right) \Gamma\left(1+t_{i}\right)}{\Gamma\left(1+\lambda_{i}\right)}, \\
D_{i} & =D_{i-1} a_{i} \Delta_{i},
\end{aligned}
$$

where $\lambda_{0}=0$ and $D_{0}=1$.

Proof. : See Proof of Lemma 1 in Appendix.

The following propositions summarize the results for the cdf and MGF of $\bar{\gamma}_{\text {MRC }}$ for high-SNR regime.

Proposition 2. Let $\bar{\gamma}_{i}, i=1, \ldots, M$, be independent branch SNRs. The asymptotic cdf and MGF of the $\bar{\gamma}_{\mathrm{MRC}}$ in generalized fading environments are, respectively, given by

$$
\begin{aligned}
P_{\bar{\gamma}_{\mathrm{MRC}}}(\gamma) \approx \sum_{j_{1}=1}^{N_{1}} \cdots \sum_{j_{M}=1}^{N_{M}} \frac{1}{\Gamma\left(\sum_{i=1}^{M} v_{i j_{i}} m_{i j_{i}}+1\right)} \\
\times \prod_{i=1}^{M} \frac{\Gamma\left(v_{i j_{i}} m_{i j_{i}}+1\right)}{\Gamma\left(m_{i j_{i}}+1\right)} \\
\times\left(\frac{\Gamma\left(m_{i j_{i}}+1 / v_{i j_{i}}\right)}{\Gamma\left(m_{i j_{i}}+1\right)} \frac{\gamma}{\bar{\gamma}_{i j_{i}}}\right)^{v_{i j_{i}} m_{i j_{i}}}
\end{aligned}
$$

$$
\begin{aligned}
\mathcal{M}_{\bar{\gamma}_{\mathrm{MRC}}}(s)= & \prod_{i=1}^{M} \mathcal{M}_{\gamma_{i}}(s) \\
\approx & \sum_{j_{1}=1}^{N_{1}} \ldots \sum_{j_{M}=1}^{N_{M}} \prod_{i=1}^{M} \frac{\Gamma\left(v_{i j_{i}} m_{i j_{i}}+1\right)}{\Gamma\left(m_{i j_{i}}+1\right)} \\
& \times\left(\frac{\Gamma\left(m_{i j_{i}}+1 / v_{i j_{i}}\right)}{\Gamma\left(m_{i j_{i}}\right)} \frac{1}{\bar{\gamma}_{i j_{i}} s}\right)^{v_{i j_{i}} m_{i j_{i}}} .
\end{aligned}
$$

Proof. : Using Lemma 1 and employing the result for the product of two series [27, Eq. (10)] gives, after some manipulation, the desired result in (16a).

The MGF of $\bar{\gamma}_{\text {MRC }}$ can directly be found from

$$
\mathcal{M}_{\bar{\gamma}_{\text {MRC }}}(s)=s \mathcal{L}\left(P_{\bar{\gamma}_{\text {MRC }}}(\gamma)\right)
$$

where $\mathcal{L}(\cdot)$ denotes the Laplace transform. Therefore, substituting the cdf given in (16a) into (17) and using $\mathcal{L}\left(x^{\nu}\right)=$ $\Gamma(v+1) / s^{\nu+1}$, the MGF given in (16b) is achieved.

\subsubsection{SC}

Instead of using MRC, which requires exact knowledge of the all CSIs, a system may use SC which simply requires SNR measurements. Indeed, SC is considered as the least complicated receiver $[26, \mathrm{Ch} .11]$. The total SNR at the output of the $\mathrm{SC}$ combiner is given by

$$
\gamma_{\mathrm{SC}} \leq \bar{\gamma}_{\mathrm{SC}}=\max \left(\bar{\gamma}_{1}, \ldots, \bar{\gamma}_{M}\right)
$$
$\bar{\gamma}_{S C}$.

The following proposition gives the cdf and the MGF of

Proposition 3. Let $\bar{\gamma}_{i}, i=1, \ldots, M$ be independent branch SNRs. The asymptotic cdf and the MGF of the $\bar{\gamma}_{\mathrm{SC}}$ in generalized fading environments are, respectively, given by

$$
\begin{aligned}
P_{\bar{\gamma}_{\mathrm{SC}}}(\gamma) \approx & \sum_{j_{1}=1}^{N_{1}} \cdots \sum_{j_{M}=1}^{N_{M}} \prod_{i=1}^{M} \frac{1}{\Gamma\left(m_{i j_{i}}+1\right)} \\
& \times\left(\frac{\Gamma\left(m_{i j_{i}}+1 / v_{i j_{i}}\right)}{\Gamma\left(m_{i j_{i}}\right)} \frac{\gamma}{\bar{\gamma}_{i j_{i}}}\right)^{v_{i j} m_{i j}}, \\
\mathcal{M}_{\overline{\gamma_{\mathrm{SC}}}}(s) \approx & \sum_{j_{1}=1}^{N_{1}} \ldots \sum_{j_{M}=1}^{N_{M}} \Gamma\left(1+\sum_{i=1}^{M} v_{i j_{i}} m_{i j_{i}}\right) \prod_{i=1}^{M} \frac{1}{\Gamma\left(m_{i j_{i}}+1\right)} \\
& \times\left(\frac{\Gamma\left(m_{i j_{i}}+1 / v_{i j_{i}}\right)}{\Gamma\left(m_{i j_{i}}\right)} \frac{1}{\bar{\gamma}_{i j_{i}} s}\right)^{v_{i j_{i}} m_{i j i}}
\end{aligned}
$$


Proof. : If the branches fade independently, the cdf of the $\bar{\gamma}_{\mathrm{SC}}$ is given

$$
\begin{aligned}
P_{\overline{\gamma_{S C}}}(\gamma)= & \operatorname{Pr}\left(\gamma_{i}<\gamma, 1 \leq i \leq M\right) \\
= & \prod_{i=1}^{M} P_{\overline{\gamma_{i}}}(\gamma)=\prod_{i=1}^{M} \sum_{j=1}^{N_{i}} \frac{1}{\Gamma\left(m_{i j}+1\right)} \\
& \times\left(\frac{\Gamma\left(m_{i j}+1 / v_{i j}\right)}{\Gamma\left(m_{i j}\right)}\right)^{v_{i j} m_{i j}}\left(\frac{\gamma}{\bar{\gamma}_{i j}}\right)^{v_{i j} m_{i j}} .
\end{aligned}
$$

Using the result in [27, Eq. (10)] for the product of two series, the desired result given in $(21 \mathrm{~b})$ is derived.

The MGF of $\bar{\gamma}_{\text {SC }}$ can be obtained following the same procedure used to obtain (16b).

\subsection{Performance analysis}

In this section, the outage probability and the SER are derived for the MRC and SC receivers.

\subsubsection{Outage probability}

Using (16a) and (21b), the asymptotic outage probability of the system with MRC and SC receivers can readily be obtained as

$$
\begin{aligned}
P_{\mathrm{out}}^{\mathrm{MRC}} \approx & \sum_{j_{1}=1}^{N_{1}} \cdots \sum_{j_{M}=1}^{N_{M}} \frac{1}{\Gamma\left(\sum_{i=1}^{M} v_{i j_{i}} m_{i j_{i}}+1\right)} \prod_{i=1}^{M} \\
& \frac{\Gamma\left(v_{i j_{i}} m_{i j_{i}}+1\right)}{\Gamma\left(m_{i j_{i}}+1\right)}\left(\frac{\Gamma\left(m_{i j_{i}}+1 / v_{i j_{i}}\right)}{\Gamma\left(m_{i j_{i}}+1\right)} \frac{\gamma_{t h}}{\bar{\gamma}_{i j_{i}}}\right)^{v_{i j_{i}} m_{i j_{i}}},
\end{aligned}
$$

$$
\begin{aligned}
P_{\mathrm{out}}^{\mathrm{SC}} \approx & \sum_{j_{1}=1}^{N_{1}} \ldots \sum_{j_{M}=1}^{N_{M}} \prod_{i=1}^{M} \frac{1}{\Gamma\left(m_{i j_{i}}+1\right)} \\
& \times\left(\frac{\Gamma\left(m_{i j_{i}}+1 / v_{i j_{i}}\right)}{\Gamma\left(m_{i j_{i}}\right)} \frac{\gamma_{t h}}{\bar{\gamma}_{i j_{i}}}\right)^{v_{i j_{i}} m_{i j_{i}}},
\end{aligned}
$$

\subsubsection{SER}

Note that the asymptotic SER is found by substituting our results for the asymptotic MGF in (16b) and (19b) into (12) and (13), respectively, for the MRC and SC receiver with $M$-PSK and $M$-QAM. However, seeking a closed-form solution to (12) and (13) is intractable due to the integration over $\theta$. To avoid this integration, we invoke the accurate approximations in [28, Eq. (34)] and [28, Eq. (36)] to get the asymptotic SER for $M$-PSK and $M$-QAM, respectively.

\subsubsection{Diversity order}

By defining the diversity order as $d=\lim _{\bar{\gamma} \rightarrow \infty}-$ $\log \left(P_{\text {out }}\right) / \log (\bar{\gamma})$, one can easily check that MRC and SC receiver attain diversity order

$$
d=\sum_{i=1}^{M} \min \left(v_{i 1} m_{i 1}, \ldots, v_{i N_{i}} m_{i N_{i}}\right)
$$

Note that although both MRC and SC schemes achieve the same diversity order, the MRC scheme achieves an additional coding gain.

\section{Power allocation for multi-hop multi-branch cooperative system}

In this section, two effective transmit power allocation schemes are described. The power allocation scheme which tends to minimize the total power of the system is developed in Section 5.2. A suboptimal scheme is proposed in Section 5.3 aimed at minimizing the outage probability. In the sequel, a brief introduction of GP for application to be discussed in the next two sections on power control problems is given.

\subsection{GP}

GP is well-investigated class of nonlinear, non-convex optimization problems, which can be turned into a convex optimization problem [18]. Hence, a local optimum of a GP problem is also a global optimum, which can always be calculated efficiently using interior-point methods [29]. The polynomial time complexity of the interior-point methods, their high speed in practice, and availability of large-scale software solvers make GP more appealing ${ }^{\mathrm{e}}$ (please see GP in Appendix for details on GP). We show that the corresponding optimization problems can be formulated as GP and thus optimal power allocation (OPA) can be obtained using the convex optimization techniques.

\subsection{Minimizing the total transmit power}

We consider the problem of minimizing the total transmitter power subject to bounds on individual powers and outage constraint. Note that to improve the system performance, the transmitting nodes can transmit at their maximum available power which cause themselves to run out of energy rapidly. This also implies that the number of available relay nodes will decrease quickly, which leads to lower throughput and higher transmission power for each node. However, by considering the QoS requirements, the channel qualities and OPA at the source and relay nodes, some of the transmitting nodes save their power and prolong their lifetime. In order to minimize the total transmit power of all nodes, subject to constraints on 
the individual transmitter powers and subject to a maximum allowed outage probability $\epsilon$ at the destination, we form the optimization problem as $\mathrm{f}$

$$
\begin{aligned}
\operatorname{minimize} & \sum_{i=1}^{M} \sum_{j=0}^{N_{i}-1} P_{i j}, \\
\text { subject to } & P_{i j}^{\min } \leq P_{i j} \leq P_{i j}^{\max }, \quad i=1, \ldots, M, \\
& \\
& P_{\text {out }}^{\mathrm{MRC}} \leq \epsilon,
\end{aligned}
$$

where $P_{i 0}=P_{0}, i=1, \ldots, M$ is the source power; $P_{i j}^{\min }$ and $P_{i j}^{\max }$ are, respectively, the minimum and maximum transmission power for the corresponding node which can be the same or different for rely nodes. Note that $P_{\text {out }}^{\mathrm{MRC}}$ in (23) is a nonlinear function of the powers, which yields a posynomial upper bound inequality constraint for the optimization problem in (23). With MRC receiver at the destination, the optimization problem in (23) can be expressed as

$$
\begin{array}{ll}
\operatorname{minimize} & \sum_{i=1}^{M} \sum_{j=0}^{N_{i}-1} P_{i j}, \\
\text { subject to } & \frac{P_{i j}^{\min }}{P_{i j}} \leq 1, \quad i=1, \ldots, M \\
& \frac{P_{i j}}{P_{i j}^{\max } \leq 1, \quad i=0, \ldots, N_{i}-1, \ldots, M,} \\
& \frac{1}{\epsilon} \sum_{j_{1}=1}^{N_{1}} \ldots \sum_{j_{M}=1}^{N_{M}} \frac{j}{\Gamma\left(\sum_{i=1}^{M} v_{i j_{i}} m_{i j_{i}}+1\right)} \\
& \times\left(\frac{\gamma_{t h}}{\bar{\gamma}_{i j_{i}}}\right)^{\sum_{i=1}^{M} v_{i j_{i}} m_{i j_{i}}} \prod_{i=1}^{M} \psi_{i j_{i}} \leq 1,
\end{array}
$$

where $\psi_{i j_{i}}=\frac{\Gamma\left(v_{i j} m_{i j_{i}}+1\right)}{\Gamma\left(m_{i j_{i}}+1\right)}\left(\frac{\Gamma\left(m_{i j i}+1 / v_{i j_{i}}\right)}{\Gamma\left(m_{i j_{i}}+1\right)}\right)^{v_{i j_{i}} m_{i j_{i}}}$. Each of the terms $\bar{\gamma}_{i j_{i}}$ is a posynomial in $P_{i j}$ and the product of posynomials is also a posynomial [18]. Moreover, the inequality constraints (24b) and (24c) are monomial and the constraint in (24d) is a posynomial. Therefore, the optimization problem in (24) is a GP in the variables $P_{i j}, i=1, \ldots, M, j=0, \ldots, N_{i}-1$. By using the interiorpoint methods for GP we can solve the power allocation problem in (24).

\subsection{Minimizing the outage probability}

In this section, we explore the power allocation policy aimed at minimizing the outage probability. The problem formulation (24) can readily be modified to minimize the asymptotic outage probability as

$$
\begin{array}{cl}
\text { minimize } & P_{\text {out }}^{\mathrm{MRC}} \\
\text { subject to } & \frac{1}{P_{T}} \sum_{i=1}^{M} \sum_{j=0}^{N_{i}-1} P_{i j} \leq 1
\end{array}
$$

where $P_{T}=\sum_{i=1}^{M} \sum_{j=1}^{N_{i}} P_{i j}^{\max }$ is the total available power. It is obvious that the optimization problem (25) belongs to the class of GP problems and can efficiently be solved by using the interior-point methods.

\subsubsection{Analytical results for a single-relay cooperative network}

In this section, we provide an analytical approximation of optimum power allocation for a three-node cooperative network, with a source $S$, a relay $R$, and a destination $D$. This analysis provides some insight for the formulated problem in section 5.3. We denote by $P_{1}$ and $P_{2}$ the transmitted power from source and relay, respectively. The optimization problem in (25) is then given by

$$
\begin{array}{cl}
\operatorname{minimize} & \frac{C_{1}}{P_{1}^{m_{S D} v_{S D}} P_{1}^{m_{S R} v_{S R}}}+\frac{C_{2}}{P_{1}^{m_{S D} v_{S D}} P_{2}^{m_{R D} v_{R D}}} \\
\text { subject to } & P_{1}+P_{2} \leq P_{T},
\end{array}
$$

where $C_{1}$ and $C_{2}$ are positive constants, capturing the fading effects of the links. Since the fading parameters generally take non-integer values, solving (26) does not yield closed-form expressions for $P_{1}$ and $P_{2}$. Nevertheless, the optimization problem defined in (26) includes, as special case, the Rayleigh fading environment. In this case, the power allocation problem is reduced to

$$
\begin{array}{cl}
\text { minimize } & \frac{1}{P_{1}}\left(\frac{1}{P 1}+\frac{\kappa}{P_{2}}\right) \\
\text { subject to } & P_{1}+P_{2} \leq P_{T},
\end{array}
$$

where $\kappa=\frac{\Omega_{S R}}{\Omega_{R D}}$, with $\Omega_{S R}=\mathbb{E}\left(\left|h_{S R}\right|^{2}\right)$ and $\Omega_{R D}=$ $\mathbb{E}\left(\left|h_{R D}\right|^{2}\right)$, is a measure for the quality of the S-R link compared to R-D link. Denoting the optimal source and relay powers by $P_{1}^{*}$ and $P_{2}^{*}$, respectively, and defining, $\alpha^{*}=\frac{P_{1}^{*}}{P_{2}^{*}}$, the OPA can be obtained from (27) as $P_{1}^{*}=P_{T} /\left(1+\alpha^{*}\right)$ and $P_{2}^{*}=\alpha^{*} P_{T} /\left(1+\alpha^{*}\right)$, with

$$
\alpha^{*}=\frac{1}{4}\left(-\kappa+\sqrt{\kappa^{2}+8 \kappa}\right) .
$$

From (28) we observe that

1. When the relay is close to the destination, optimum value of $P_{1}$ is $\sim P_{T}$, and that of $P_{2}$ is $\sim 0$. These 
values indicate that it is better to spend most of the power in broadcast phase.

2. When the relay is located midway between the source and destination, optimum value of $P_{1}$ is $\sim(2 / 3) P_{T}$ which means that $66 \%$ of power should be spent in the broadcast phase and $33 \%$ of power should be dedicated to the relay terminal in the relaying phase. These values indicate that it is better to spend most of the power in broadcast phase.

3. when relay is close to the source, $P_{1}$ and $P_{2}$ are found to be $\sim 0.5 P_{T}$ indicating that equal power allocation (EPA) is nearly optimal.

Note that the same observations have been reported in $[30,31]$ for a three-node cooperative network.

\subsection{Discussion on the implementation of power allocation schemes}

The two proposed power allocation schemes are computed in a centralized manner at the destination. Centralized implementation of power allocation schemes requires a central controller to collect the information of all wireless links in order to find an optimal solution, and distribute the solution to the corresponding wireless nodes. Hence, information exchange plays a crucial role in implementing the resource optimization process. Useful information can be the full channel state information, or partial channel state information (e.g., average channel realizations), or some other quantized/codebook-based limited-rate feedback information.
The implementation of our proposed power allocation schemes requires that the destination has the information about the channel statistics rather than the instantaneous CSIs. Since the first-order and secondorder statistics vary much slower than the instantaneous CSIs, the overhead is significantly reduced. The remaining, but most challenging task is keeping the amount of feedback overhead information, exchanged within the network, at a reasonable level. For this purpose, the destination determines the power coefficients. These coefficients are then quantized at the receiver and sent back to the transmitters over a low-rate feedback link [32]. Therefore, the signaling overhead is much lower than that of the conventional centralized methods.

\section{Simulation results}

In this section, we provide numerical results corroborating the analysis developed in the previous sections. It is assumed that the relays and the destination have the same value of noise power. We plot the performance curves in terms of outage probability and average SER versus the normalized average SNR per hop. We also set $\gamma_{\text {th }}=3 \mathrm{~dB}$.

Figure 2 plots the exact and asymptotic outage probability of a generic cooperative system in the context of various scenarios. Specifically, the outage probability of two and three branches AF network have been plotted for MRC and SC receivers. Moreover, the outage performance of direct transmission is also depicted as the baseline for comparison. In our simulations, the

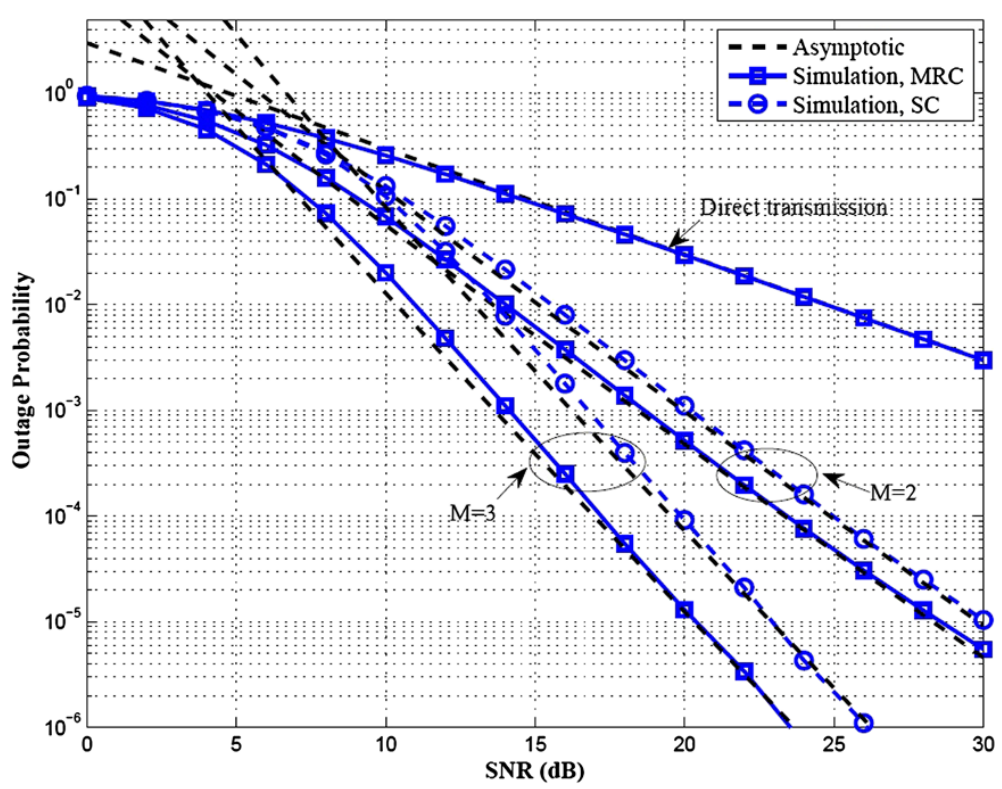

Figure 2 Outage probability. Outage probability for one, two, and three branches AF relay network. (System configuration: First branch: Rayleigh fading, Second branch: Nakagami- $m\left(m_{21}=1.1\right)$ and Nakagami- $m\left(m_{22}=1.6\right)$, Third branch: Rician $(K=5 \mathrm{~dB})$, Nakagami- $m\left(m_{21}=0.9\right)$ and Nakagami- $m\left(m_{22}=2\right)$ 
direct channel (first branch) is assumed to be Rayleigh fading channel corresponding to $m_{11}=1$. By contrast, the dual-hop link (second branch) is assumed to be consisted of Nakagami- $m$ fading channels associated with $m_{21}=1.1$ and $m_{22}=1.6$. Moreover, the triplehop channel (third branch) is assumed to be consisted of Rician and Nakagami- $m$ fading channels associated with $K=5 \mathrm{~dB}$ and $m_{32}=0.9$ and $m_{33}=3$. The validity of our asymptotic results in (21a) and (21b) are attested to in the figure, where the asymptotic curves correctly predict the diversity order and the array gain of the exact SER.

In Figure 3, we evaluated the SER performance of the cooperative wireless systems, when assuming both BPSK and 64-QAM baseband modulation schemes. These figure also demonstrate the accuracy of the asymptotic SER evaluated with the aid of the MGF of the received SNR in comparison with the SER obtained by simulations. Note that the corresponding curves are plotted only for direct transmission and $M=3$ branches case, to avoid entanglement. The results of Figure 3 shows that the asymptotic SER is very accurate especially in high SNR region. Note that our observation of the outage performance of 8-QAM and 16-QAM modulations, which for the sake of clarity are not shown in Figure 3, reveals that the asymptotic SER matches the exact results in higher SNR values, as the modulation order increases. We interpret this behavior as a consequence of approximation, used in [28] to derive the closed-form SER expressions.
Next, we compare the performance of the optimum and EPA, with the latter equally distributing the power among all the relay nodes. Figure 4 shows the outage performance of the proposed OPA scheme in (25) in the context of various scenarios. Specifically, in our simulations, we consider $M=2$ and $M=3$ branches cases, where channels undergo the same statistical process as that in Figure 2. Curves for 16-QAM modulation are plotted for SC and MRC receivers, while corresponding curves from analysis are plotted only for $M=2$, to avoid entanglement. We observe that the power allocation shows significant improvement in performance compared to those of a system with EPA. Moreover, the gap in performance increases further with increase in SNR values and the number of branches. Note that, instead of minimizing the outage probability in (25), we can minimize the SER performance of the system. Figure 5 shows a comparison between the equal power and OPA schemes for multi-hop multi-branch system using BPSK modulation. SER Curves for SC and MRC receivers are plotted and channels undergo the same statistical process as that in Figure 2.

Figure 6 shows a comparison of the outage probability of the single-relay cooperative system for two different relay positions: relay is midway between the source and the destination, and relay is located closed to the destination. In this figure, we assume that $\mathbb{E}\left(\left|h_{S D}\right|^{2}\right)=\Omega_{S D}=1 / d_{s d}^{\alpha}$, $\mathbb{E}\left(\left|h_{S R}\right|^{2}\right)=\Omega_{S R}=1 / d_{s r}^{\alpha}$, and $\mathbb{E}\left(\left|h_{R D}\right|^{2}\right)=\Omega_{R D}=$ $1 / d_{r d}^{\alpha}$, where $d_{s d}, d_{s r}$, and $d_{r d}$ are, respectively, the sourcedestination, source-relay, and relay-destination distances

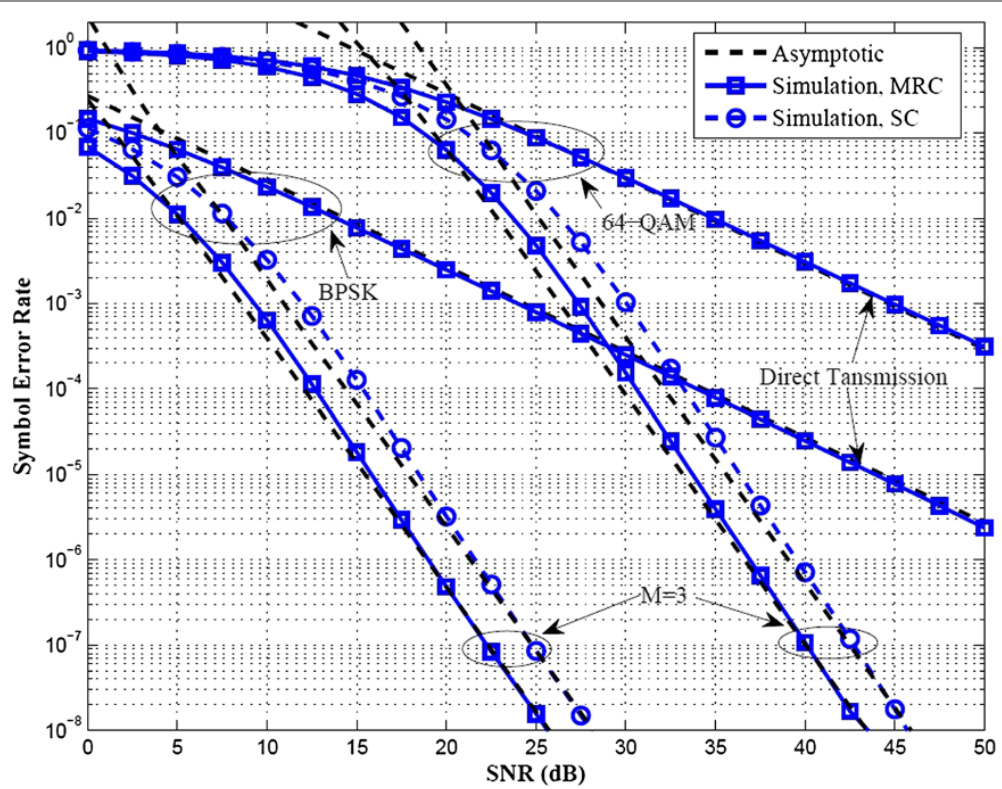

Figure 3 SER. SER versus the SNR performance of the cooperative wireless systems using BPSK and 64-QAM modulations, when direct channel experiences Rayleigh fading $\left(m_{11}=1\right)$, the dual-hop channels experience Nakagami-m fading associated with $m_{21}=1.1, m_{22}=1.6$, and the triple-hop channels experience Rician fading and Nakagami- $m$ fading associated with $K=5 \mathrm{~dB}$ and $m_{21}=0.9, m_{22}=2$, respectively. 


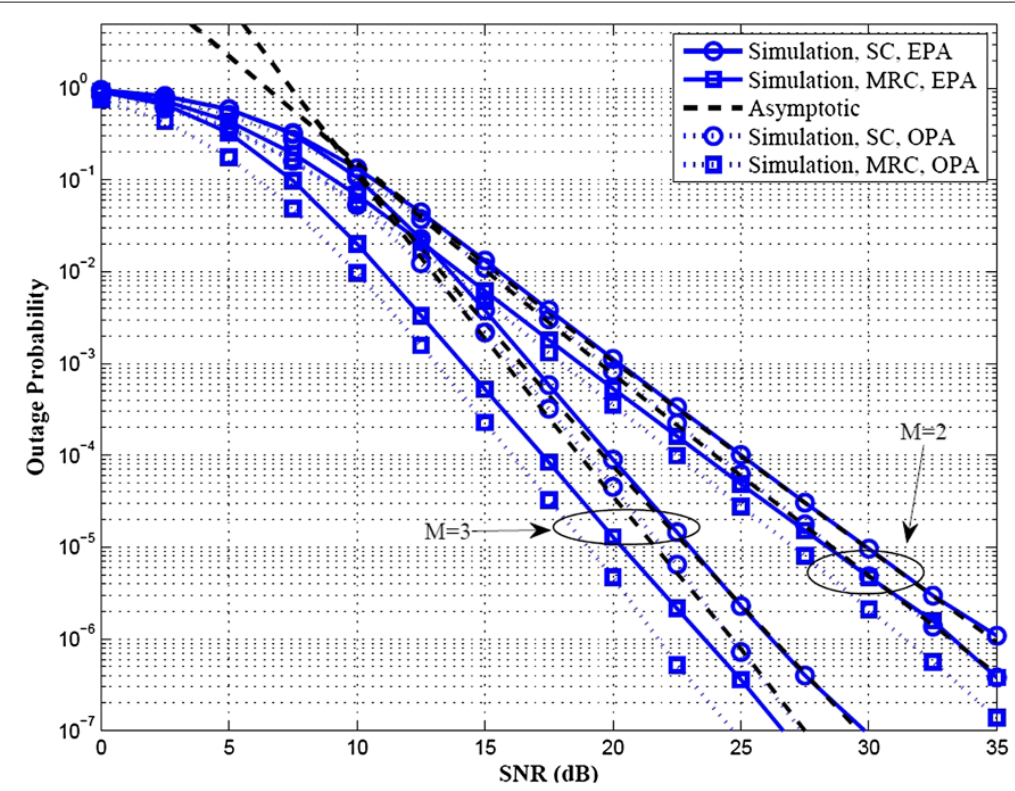

Figure 4 Outage probability for EPA and OPA. Outage probability versus the SNR performance of the cooperative wireless systems using 16-QAM modulation for two power allocation schemes: EPA and proposed power allocation in 25a. In this figure, channels undergo the same statistical process as that in Figure 2. Asymptotic bounds have been obtained using (21a) and (21b) for MRC and SC receiver, respectively.

and $\alpha$ is the path loss exponent. We also set $\alpha=3$. The solid curves are the outage probability with EPA and the dotted curves are the outage probability with OPA in (28). Clearly and as expected, for these two relay placements, EPA does not give the best performance. Moreover, the OPA is more suitable to be utilized in single-relay cooperation networks in which relay is located close to the destination.

\section{Conclusion}

We investigated the performance of multi-hop multibranch AF relay systems in generalized fading environment

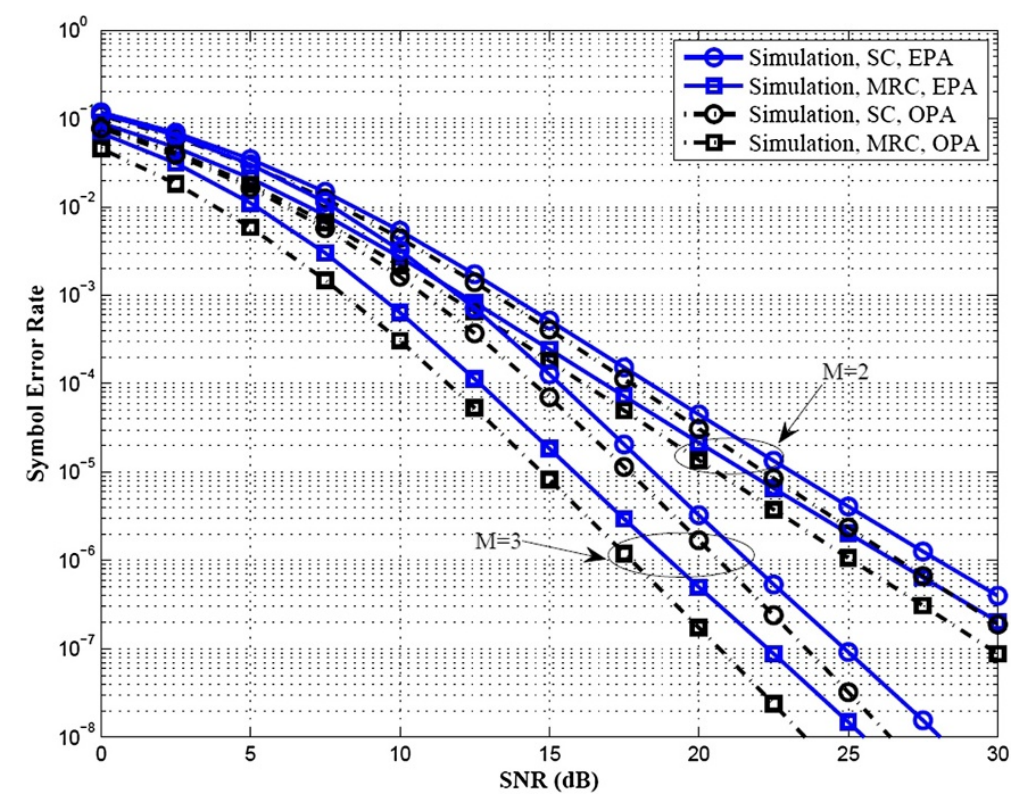

Figure 5 SER for EPA and OPA. SER versus the SNR performance of the cooperative wireless systems using BPSK modulation for two power allocation schemes: EPA; proposed power allocation in (25a). In this figure, channels undergo the same statistical process as that in Figure 2. 


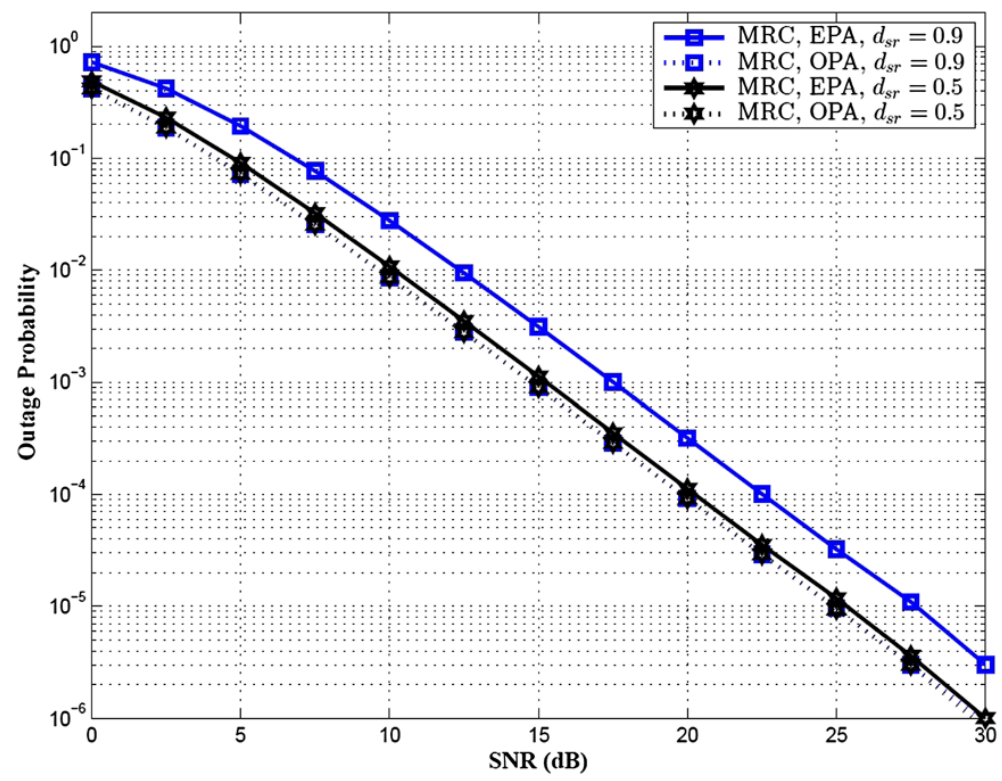

Figure 6 Comparison of the outage probability of the cooperative system using EPA and the OPA in two relay position. Comparison of the outage probability of the cooperative system for QPSK modulation using EPA and the OPA in two configurations: (1) relay is located close to the destination, (2) relay is located midway between the source and destination. In this setup both direct channel and relay channel experience Rayleigh fading.

with MRC and SC receivers. A range of closed-form results has been derived for both the statistics of the output SNR and the asymptotic performance of the system under study. We substantiated the tightness of such asymptotic expressions and the accuracy of our theoretical analysis using simulation results. Moreover, we developed two power allocation strategies for further improving the cooperation. The first strategy sought to minimize the total transmit power; the second strategy aimed at minimizing the outage probability, which was parameterized by the total power available to the relay nodes and the source node. We found that the OPA shows significant improvement in performance when relay nodes are asymmetrically placed at fixed locations when compared to a system with EPA.

\section{Endnotes}

${ }^{\text {a } W e ~ a s s u m e ~ t h a t ~ t h e ~ n o i s e ~ p o w e r ~ i s ~ i d e n t i c a l ~ i n ~ a l l ~ r e c e i v-~}$ ing nodes. Note that this assumption is not essential and can easily be relaxed, but at the cost of complicating the derived expressions without providing additional insight.

${ }^{b}$ We notice that closed-form expressions for the statistics of $\gamma_{i}$ are given in [3] and [4] for the special case of an AF dual-hop system in Nakagami- $m$ and Rayleigh fading channels, respectively.

' In [35], an accurate approximation has been presented for the SER with $M$-PSK modulation.

${ }^{\mathrm{d}}$ In this study, we assume that the receiver estimates the channel perfectly from training. A discussion of channel estimation techniques is beyond the scope of this article and the reader is referred to $[36,37]$ for the details.

${ }^{\text {e}}$ There are several high-quality software downloadable from the Internet, which are widely used to solve the GP using interior-point methods (e.g., the MOSEK package and the CVX package).

${ }^{\mathrm{f}}$ Note that we consider the MRC combiner in the proposed power allocation schemes. However, for the SC combiner, we can follow the same procedure to get the optimized transmitted powers.

\section{Appendix}

\section{Proof of Proposition 1}

Assuming that the received SNR's from different diversity branches are independent, the cdf of the received SNR in (6) is given by

$$
\begin{array}{r}
P_{\gamma_{i}}(\gamma)=\operatorname{Pr}\left(\min \left(\gamma_{i 1}, \ldots, \gamma_{i N_{i}}\right) \leq \gamma\right) \\
\stackrel{(a)}{=} 1-\prod_{j=1}^{N_{i}} \frac{\Gamma\left(m_{i j},\left(\frac{\xi_{i j}}{\bar{\gamma}_{i j}} \gamma\right)^{v_{i j}}\right)}{\Gamma\left(m_{i j}\right)},
\end{array}
$$

where $(a)$ follows by using the complementary cdf of the generalized Gamma distribution. With the help of [22, Eq. (8.356.4)] the cdf can be rewritten as 


$$
\begin{aligned}
P_{\gamma_{i}}(\gamma)=1- & \prod_{j=1}^{N_{i}}\left(1-\sum_{k=0}^{\infty} \frac{(-1)^{k}}{k !\left(m_{i j}+k\right) \Gamma\left(m_{i j}\right)}\right. \\
& \left.\left(\frac{\xi_{i j}}{\bar{\gamma}_{i j}} \gamma\right)^{v_{i j} m_{i j}+k}\right) .
\end{aligned}
$$

Using the inequality $1+\sum_{\ell=1}^{K} z_{\ell} \leq \prod_{\ell=1}^{K}\left(1+z_{\ell}\right) \leq$ $\exp \left(\sum_{\ell=1}^{K} z_{\ell}\right)$ [33, Appendix II] we get

$$
\begin{aligned}
& \sum_{j=1}^{N_{i}} \sum_{k=0}^{\infty} \frac{(-1)^{k}}{k !\left(m_{i j}+k\right) \Gamma\left(m_{i j}\right)}\left(\frac{\xi_{i j}}{\bar{\gamma}_{i j}} \gamma\right)^{v_{i j} m_{i j}+k} \leq P_{\gamma_{i}}(\gamma) \leq \\
& 1-\exp \left(\sum_{k=0}^{\infty} \frac{(-1)^{k}}{k !\left(m_{i j}+k\right) \Gamma\left(m_{i j}\right)}\left(\frac{\xi_{i j}}{\bar{\gamma}_{i j}} \gamma\right)^{v_{i j} m_{i j}+k}\right) .
\end{aligned}
$$

By approximating the exponential term in the righthand side of the inequality with the first two terms of the well- known Taylor series, for high SNRs $\left(1 / \bar{\gamma}_{i j} \rightarrow 0\right)$, the cdf in (31) is further simplified as

$$
P_{\gamma_{i}}(\gamma) \approx \sum_{j=1}^{N_{i}} \sum_{k=0}^{\infty} \frac{(-1)^{k}}{k !\left(m_{i j}+k\right) \Gamma\left(m_{i j}\right)}\left(\frac{\xi_{i j}}{\bar{\gamma}_{i j}} \gamma\right)^{v_{i j} m_{i j}+k} .
$$

Our simulation results in Section 6 show that for $k=0$, a fairly tight asymptotic bound for the outage probability of the multi-hop system is achieved. The reason is that the outage probability is proportional to $1 /\left(\bar{\gamma}_{i j}\right)^{k}$ and thus for sufficiently high values of SNRs decays very fast with $k \geq$ 1 . Therefore, substituting $\xi_{i j}$ into (32), setting $k=0$, and using the fact that $\Gamma(1+z)=z \Gamma(z)$ [34] the desired result in (8) is achieved.

\section{Proof of Lemma 1}

Let we define

$$
\begin{aligned}
\Phi_{k} \triangleq \int_{0}^{w}\left(w-x_{k}\right)^{\lambda_{k-1}} x_{k}^{\nu_{k}} d x_{k} \stackrel{(a)}{=} & \frac{\Gamma\left(1+\lambda_{k-1}\right) \Gamma\left(1+v_{k}\right)}{\Gamma\left(1+\lambda_{k}\right)} w^{\lambda_{k}} \\
& =\Delta_{k} w^{\lambda_{k}},
\end{aligned}
$$

where ( $a$ ) follows by using [22], $\lambda_{k}:=\lambda_{k-1}+v_{k}+1$, and $\Delta_{k}$ is defined in (15d). To prove the proposition, we will use mathematical induction. Clearly, Lemma 1 holds for $n=1$ and $n=2$, i.e.,

$$
P_{z_{1}}(w) \stackrel{(a)}{=} \frac{c_{1}}{v_{1}+1} w^{v_{1}+1}=c_{1} \Phi_{1}=D_{1} w^{\lambda_{1}}
$$

$$
\begin{aligned}
P_{z_{2}}(w) & =\int_{0}^{w} \operatorname{Pr}\left(x_{1}<w-x_{2}\right) f_{x_{2}} d x_{2} \\
& \stackrel{(b)}{=} c_{1} c_{2} \Delta_{1} \int_{0}^{w}\left(w-x_{2}\right)^{\nu_{1}+1} x_{2}^{\nu_{2}} d x_{2}=c_{1} c_{2} \Delta_{1} \Phi_{2} \\
& =D_{2} w^{\lambda_{2}},
\end{aligned}
$$

where $(a)$ follows by taking the integral of the pdf of $z_{1}=$ $x_{1}$ and $(b)$ follows by using the cdf of the $z_{1}$. Suppose the result in (15a) is true for $2<n=(k-1)<M$, then for $n=k$ we have

$$
\begin{aligned}
P_{z_{k}}(w) & =\int_{0}^{w} \operatorname{Pr}\left(z_{k-1}<w-x_{k}\right) f_{x_{k}}\left(x_{k}\right) d x_{k} \\
& \stackrel{(a)}{=} c_{k} D_{k-1} \int_{0}^{w}\left(w-x_{k}\right)^{\lambda_{k-1}} x_{k}^{\nu_{k}} d x_{k}=c_{k} D_{k-1} \Delta_{k} w^{\lambda_{k}} \\
& =D_{k} w^{\lambda_{k}},
\end{aligned}
$$

where (a) follows by induction assumption. Therefore, the closed-form cdf in (15a) is valid for $n=k$, which completes the proof.

\section{GP}

In this section, we give a brief review of the GP and refer the reader to [18, Ch. 4] for details.

Let $x_{1}, \ldots, x_{n}$ be $n$ real positive variables and $x$ denotes the vector of $n$ variables. We define a monomial as a function of $f: \mathcal{R}_{++}^{n} \rightarrow \mathcal{R}:$

$$
f(x)=c x_{1}^{\alpha_{1}} x_{2}^{\alpha_{2}} \cdots x_{n}^{\alpha_{n}},
$$

where $c>0$ and $\alpha_{\ell} \in \mathcal{R}, \ell=1,2, \ldots, n$. A sum of monomials is called a posynomial function, which has the form

$$
f(x)=\sum_{k=1}^{K} c_{k} x_{1}^{\alpha_{1 k}} x_{2}^{\alpha_{2 k}} \cdots x_{n}^{\alpha_{n k}},
$$

where $c_{k} \geq 0$ and $\alpha_{k} \in \mathcal{R}, \ell=1,2, \ldots, n, k=$ $1,2, \ldots, K$.

Minimizing a posynomial subject to posynomial upper bound inequality constraints and monomial equality constraints is called GP. Therefore, a GP in standard form in $x=\left[x_{1}, \ldots, x_{n}\right]$ is given as

$$
\begin{array}{cl}
\operatorname{minimize} & f_{0}(x), \\
\text { subject to } & f_{i}(x) \leq 1 \quad i=1, \ldots, m, \\
& g_{i}(x)=1, \quad i=1, \ldots, p \\
& x_{i} \geq 0, \quad i=1, \ldots, n .
\end{array}
$$


With a logarithmic change of variables as $y_{i}=\log x_{i}$ (or equivalently $x_{i}=\exp \left(y_{i}\right)$ which enforces the positivity constraint on $x_{i}$ ) we can turn the GP in (38) into the following equivalent problem in $x$

$$
\begin{aligned}
\operatorname{minimize} & \log f_{0}\left(\exp \left(y_{1}\right), \ldots, \exp \left(y_{n}\right)\right), \\
\text { subject to } & \log f_{i}\left(\exp \left(y_{1}\right), \ldots, \exp \left(y_{n}\right)\right) \leq 1 \quad i=1, \ldots, m, \\
& \log g_{i}\left(\exp \left(y_{1}\right), \ldots, \exp \left(y_{n}\right)\right)=1, \quad i=1, \ldots, p,
\end{aligned}
$$

which is a convex problem, since the objective function and the inequality constraint functions are all convex and the equality constraint functions are affine (note that the log-sum-exp function is convex [18]).

\section{Competing interests}

The author declare that they have no competing interests.

\section{Acknowledgements}

This work is supported in part by the Ministry of Industries and Mines of Iran.

Received: 10 June 2012 Accepted: 12 February 2013

Published: 12 June 2013

\section{References}

1. R Pabst, B Walke, D Schultz, P Herhold, H Yanikomeroglu, S Mukherjee, H Viswanathan, M Lott, W Zirwas, M Dohler, H Aghvamia, D Falconer, GP Fettweis, Relay-based deployment concepts for wireless and mobile broadband radio. IEEE Commun. Mag. 42, 80-89 (2004)

2. E Morgado, I Mora-Jiménez, JJ Vinagre, J Ramos, AJ Caamaño, End-to-end average BER in multihop wireless networks over fading channels. IEEE Trans. Wirel. Commun. 9(8), 2478-2487 (2010)

3. MO Hasna, MS Alouini, Harmonic mean and end-to-end performance of transmission systems with relays. IEEE Trans. Commun. 52(1), 130-135 (2004)

4. MO Hasna, M-S Alouini, End-to-end performance of transmission systems with relays over Rayleigh-fading channels. IEEE Trans. Wirel. Commun. 2(6), 1126-1131 (2003)

5. TA Tsiftsis, GK Karagiannidis, PT Mathiopoulos, SA Kotsopoulos, Nonregenerative dual-hop cooperative links with selection diversity. EURASIP J. Wirel. Commun. Netw. 2006, 1-8 (2006)

6. MD Renzo, F Graziosi, F Santucci, A comprehensive framework for performance analysis of dual-hop cooperative wireless systems with fixed-gain relays over generalized fading channels. IEEE Trans. Wirel. Commun. 8(10), 5060-5074 (2009)

7. MD Renzo, F Graziosi, F Santucci, Channel capacity over generalized fading channels: a novel MGF-based approach for performance analysis and design of wireless communication systems. IEEE Trans. Veh. Technol. 59(1), 127-149 (2010)

8. C Conne, M-C Ju, Z Yi, H-K Song, I-M Kim, SER analysis and PDF derivation for multi-hop amplify-and-forward relay systems. IEEE Trans. Commun. 58(8), 2413-2424 (2010)

9. HQ Huynh, SI Husain, J Yuan, A Razi, H Suzuki, Performance analysis of multibranch dual-hop nonregenerative relay systems with EGC in Nakagami-m channels. EURASIP J. Wirel. Commun. Netw. 2010 (2010)

10. F Yilmaz, M-S Alouini, A unified MGF-based capacity analysis of diversity combiners over generalized fading channels. IEEE Trans. Commun. 60(3), 862-875 (2012)

11. A Ribeiro, X Cai, GB Giannakis, Symbol error probabilities for general cooperative links. IEEE Trans. Wirel. Commun. 4(3), 1264-1273 (2005)

12. MD Renzo, F Graziosi, F Santucci, A comprehensive framework for performance analysis of cooperative multi-hop wireless systems over Log-Normal fading channels. IEEE Trans. Commun. 58(2), 531-544 (2010)

13. MD Renzo, F Graziosi, F Santucci, A unified framework for performance analysis of CSI-assisted cooperative communications over fading channels. IEEE Trans. Commun. 57(9), 2551-2557 (2009)
14. G Amarasuriya, C Tellambura, M Ardakani, Asymptotically-exact performance bounds of AF multi-hop relaying over Nakagami fading. IEEE Trans. Commun. 59(4), 962-967 (2011)

15. MO Hasna, MS Alouini, Optimal power allocation for relayed transmissions over Rayleigh-fading channels. IEEE Trans. Wirel. Commun 3(6), 1999-2004 (2004)

16. R Annavajjala, PC Cosman, LB Milstein, Statistical channel knowledge-based optimum power allocation for relaying protocols in the high SNR regime. IEEE J. Sel. Areas Commun. 25(2), 292-305 (2007)

17. G Farhadi, NC Beaulieu, Power-optimized amplify-and-forward multi-hop relaying systems. IEEE Trans. Wirel. Commun. 8(9), 4634-4643 (2009)

18. HS Boyd, L Vandenberghe, Convex Optimization. (Cambridge University Press, Cambridge, MA, 2004)

19. M Chiang, C-W Tan, DP Palomar, D O'Neill, D Julian, Power control by geometric programming. IEEE Trans. Wirel. Commun. 6(7), 2640-2651 (2007)

20. MO Hasna, M-S Alouini, Outage probability of multihop transmission over Nakagami fading channels. IEEE Commun. Lett. 7(5), 216-218 (2003)

21. MD Yacoub, in Proceedings of the IEEE PIMRC. The $\alpha-\mu$ distribution: a general fading distribution (Portugal, September 2002), pp. 629-633

22. IS Gradshteyn, IM Ryzhik, Table of Integrals, Series and Products, 7th edn. (Academic Press, New York, 2007)

23. R Vaughan, JB Andersen, Channels, Propagation and Antennasfor Mobile Communications. (IEE, London, UK, 2003)

24. S Ikki, MB Ahmed, in Proceedings of the IEEE GLOBECOM 2007. Performance analysis of dual-hop relaying communications over generalized Gamma fading channels (Washington, DC, USA, November 2007), pp. 3888-3893

25. MO Hasna, in Proceedings of the 10th IEEE International Conference on Electronics, Circuits, and Systems, vol. 2. Average BER of multihop communication systems over fading channels, (December 2003), pp. $723-726$

26. MK Simon, MS Alouini, Digital Communication over Fading Channels, 2nd edn. (Wiley, New York, NY, 2005)

27. TQ Duong, VNQ Bao, H-J Zepernick, On the performance of selection decode-and-forward relay networks over Nakagami-m fading channels. IEEE Commun. Lett. 13(3), 172-174 (2009)

28. N Yang, PL Yeoh, M Elkashlan, IB Collingsc, Z Chen, Two-way relaying with multi-antenna sources: beamforming and antenna selection. IEEE Trans. Veh. Technol. 61(6), 3996-4008 (2012)

29. Y Nesterov, Nemirovsky A, in Studies in Applied Mathematics, vol. 13. Interior-point polynomial methods in convex programming (SIAM Philadelphia, PA, 1994)

30. H Ilhan, I Altunbas, M Uysal, in Proceedings of the IEEE VTC Spring. Cooperative diversity for relay-assisted inter-vehicular communication (Singapore, May 2008), pp. 605-609

31. MM Fareed, M Uysal, BER-optimized power allocation for fading relay channels. IEEE Trans. Wirel. Commun. 7(6), 2350-2359 (2008)

32. DJ Love, Heath Jr. R W, VKN Lau, D Gesbert, BD Rao, M Andrews, An overview of limited feedback in wireless communication systems. IEEE J. Sel. Areas Commun. 26(8), 1341-1365 (2008)

33. S Kandukuri, S Boyd, Optimal power control in interference-limited fading wireless channels with outage-probability specifications. IEEE Trans. Wirel. Commun. 1(1), 46-55 (2002)

34. M Abramowitz, IA Stegun, Handbook of Mathematical Functions With Formulas, Graphs, and Mathematical Tables, 9th edn. (Dover, New York, 1970)

35. MR McKay, A Zanella, IB Collings, M Chiani, Error probability and SINR analysis of optimum combining in Rician fading. IEEE Trans. Commun. 57(3), 676-687 (2009)

36. CS Patel, GL Stüber, Channel estimation for amplify and forward relay based cooperation diversity systems. IEEE Trans. Wirel. Commun. 6(6), 2348-2356 (2007)

37. G Huang, Y Wang, J Coon, in IEEE PacRim. Performance of multi-hop decode-and-forward and amplify-and-forward relay networks with channe estimation, (August 2011), pp. 352-357

doi:10.1186/1687-1499-2013-160

Cite this article as: Mohammadi et al:: Performance analysis and power allocation for multi-hop multi-branch amplify-and-forward cooperative networks over generalized fading channels. EURASIP Journal on Wireless Communications and Networking 2013 2013:160. 